\title{
As Adoções Necessárias no Contexto Brasileiro: Características, Desafios e Visibilidade
}

\author{
Camila Aparecida Peres Borges ${ }^{1}$ \\ Fabio Scorsolini-Comin ${ }^{2}$ \\ ${ }^{1}$ Universidade Federal do Triângulo Mineiro, Uberaba-MG \\ ${ }^{2}$ Universidade de São Paulo, Ribeirão Preto-SP
}

\begin{abstract}
Resumo
As adoções necessárias envolvem casos de crianças com mais de dois anos, com irmãos, com deficiência, adoecimento crônico ou inter-racial. Este estudo teve por objetivo apresentar uma revisão integrativa acerca das adoções necessárias e de como esse tema vem sendo investigado e discutido. A partir de buscas nas bases/bibliotecas LILACS, SciELO, PePSIC e PsycINFO (2009-2018), foram recuperados 35 estudos. A escassa produção internacional pode estar relacionada ao fato de que as adoções necessárias são uma categoria própria da realidade brasileira. A maior parte dos estudos priorizou como necessárias as adoções de crianças maiores de dois anos, com poucos estudos sobre crianças com deficiência, com quadro de adoecimento e com irmãos. Recomenda-se uma maior investigação em relação às adoções inter-raciais, bem como maior conscientização dos pretendentes quanto ao perfil das crianças para adoção nas instituições de acolhimento, o que deve ser acompanhado por maior visibilidade do tema no campo científico.
\end{abstract}

Palavras-chave: adoção (criança); crianças adotivas; adoção - bibliografia

\section{The Necessary Adoptions in the Brazilian Context: Characteristics, Challenges and Visibility}

\begin{abstract}
The necessary adoptions encompass cases of children over two years of age, with siblings, disabilities, chronic or interracial illness. This study aimed to present an integrative review of the necessary adoptions and how this topic has been investigated and discussed. From a search in the databases LILACS, SciELO, PePSIC and PsycINFO (2019-2018), 35 studies were retrieved. The scarce international production may be related to the fact that the necessary adoptions are a category in the Brazilian reality. Most of the studies prioritised as necessary the adoption of children children over two years of age, with few studies on children with disabilities, sickness and siblings. Further research on interracial adoptions is recommended, as well as greater awareness of the candidates to adopt regarding the profile of children for adoption in host institutions, which should be accompanied by greater visibility of the topic in the scientific field.

Keywords: adoption (child); adopted children; adoption - bibliography
\end{abstract}

Las Adopciones Necesarias en el Contexto Brasileño: Características, Desafíos y Visibilidad

\begin{abstract}
Resumen
Las adopciones necesarias abarcan casos de niños de más de dos años, con hermanos, con discapacidad, enfermedad crónica o interracial. Este estudio tuvo por objetivo presentar una revisión integradora acerca de las adopciones necesarias y de cómo ese tema viene siendo investigado y discutido. A partir de una búsqueda en las bases LILACS, SciELO, PePSIC y PsycINFO (20092018), se recuperaron 35 estudios. La escasa producción internacional puede estar relacionada a que las adopciones necesarias son una categoría propia de la realidad brasileña. La mayoría de los estudios priorizaron como necesarias las adopciones de niños mayores de dos años, con pocos estudios sobre niños con discapacidad, con cuadro de enfermedad y con hermanos. Se recomienda una mayor investigación acerca de las adopciones interraciales, así como una mayor concientización de los candidatos a adoptar en cuanto al perfil de los niños para adopción en las instituciones de acogida, lo que debe ser acompañado por una mayor visibilidad del tema en el campo científico.

Palabras clave: adopción (niño); niños adoptivos; adopción - bibliografía
\end{abstract}

A colocação em família substituta, sendo essa nacional ou internacional, é uma medida de proteção à criança ou ao adolescente e deve ser aplicada quando esses sujeitos tiverem seus direitos violados e sem garantia de se desenvolver e conviver em família (Ghirardi, 2009; Merçon-Vargas, Rosa, \& Dell'Aglio, 2015). No Brasil, o termo adoção é definido pelo Estatuto da Criança e do Adolescente (ECA) como a possibilidade de estabelecer com alguém que não se conhece, por meio da Lei, laços de filiação e paternidade legítimos
(Brasil, 1990). Tem-se propagado na sociedade a "cultura da adoção", que tem por finalidade ressaltar o que o ECA estabelece, em que a adoção tem por objetivo oferecer um lar para as crianças que não o têm, sendo uma medida protetiva, sem rotular o adotado quanto à sua condição de saúde, cor, sexo e idade (Ferreira, 2014; Souza, Monteiro, \& Brito, 2016).

No Brasil, a chamada "Nova Lei da Adoção" (NLNA) (Lei no 12.210/2009) propõe uma aproximação entre família e instituição de acolhimento, enfatiza a 
convivência familiar e comunitária, colocando a adoção como uma medida excepcional (Brasil, 2010; Silva \& Arpini, 2013). A Lei n 13.509 de 22 de novembro de 2017 e NLNA são leis brasileiras que dispõem algumas alterações em relação aos aspectos legais da adoção, dentre elas, as adoções necessárias ou adoção especial, dando prioridade a esses casos (Brasil, 2009; 2017).

De acordo com Weber (2004), no Brasil, esses tipos de adoção são escassos por se definirem como aquelas que são mais difíceis de serem realizadas, por envolverem crianças e adolescentes que são estigmatizados como "inadotáveis". Assim, a adoção de crianças maiores (anteriormente conhecida como adoção tardia, refere-se à situação a qual a criança é adotada com mais de dois anos de idade), a adoção de crianças com deficiência física, com adoecimento crônico, a adoção inter-racial e a adoção de irmãos são caracterizadas por pertencerem a esse grupo (Brasil, 2009; Weber, 2004).

No Brasil, os pretendentes fazem parte do Cadastro Nacional de Adoção após a sua habilitação na Vara da Infância e Juventude de sua comarca. Em 3 janeiro de 2018 havia 45.719 pretendentes cadastrados, sendo que 45.473 são para adoções nacionais e 246 para adoções internacionais. A quantidade de crianças/ adolescentes para adoção é de 9.400 , ou seja, uma proporção de quatro pretendentes para cada criança (http://www.cnj.jus. $\mathrm{br} / \mathrm{cnanovo} / \mathrm{pages} / \mathrm{publico} /$ index.jsf, recuperado em 7, fevereiro, 2019).

De acordo com o Conselho Nacional de Justiça (CNJ, 2013), algumas variáveis interferem nessa discrepância, já que o perfil do filho(a) desejável pelos pretendentes nem sempre se enquadra no perfil das crianças para a adoção. A idade é a variável que se destaca em relação a esse desencontro entre as preferências do pretendente e as características das crianças e adolescentes: nove em cada dez pretendentes desejam adotar uma criança de zero a cinco anos, sendo que essa faixa etária equivale a apenas nove em cada cem crianças para adoção (CNJ, 2013). Nas adoções nacionais, em relação à cor, $92,4 \%(\mathrm{n}=42.021)$ dos pretendentes desejam que a criança/adolescente seja branca, $64,52 \%$ são indiferentes quanto ao sexo, no entanto, $27,09 \%$ optam pelo sexo feminino e $37.39 \%$ aceitam adotar irmãos. Quanto a problemas de saúde, $61,81 \%(\mathrm{n}=28.108)$ dos pretendentes desejam que a criança/adolescente não tenha doenças. A porcentagem de adotantes que aceitam algum tipo de adoecimento é pequena: 5.1\% $(\mathrm{n}=2.320)$ aceitam crianças/adolescentes portadoras do vírus HIV, $6.46 \%(n=2.937)$ com deficiência física, 3,51\% $(\mathrm{n}=1.596)$ com deficiência mental e 35,14\% $(\mathrm{n}=15.977)$ com algum outro tipo de doença (http://www.cnj.jus. br/cnanovo/pages/publico/index.jsf, recuperado em 7, fevereiro, 2019).

Já o perfil dos pretendentes que buscam por adoções internacionais é o seguinte: a maioria dos postulantes aceita crianças negras $(94,72 \%)$, são indiferentes quanto ao sexo $(88,62 \%)$, aceitam adotar irmãos $(55,69 \%)$, crianças de até três anos de idade $(0,41 \%)$ e crianças com adoecimento (http://www.cnj. jus.br/cnanovo/pages/publico/index.jsf, recuperado em 7, fevereiro, 2019). A idade e a cor das crianças se destoam em comparação ao perfil dos pretendentes nacionais, demonstrando uma maior flexibilidade dos pretendentes internacionais ao determinarem $\mathrm{O}$ perfil do(a) filho(a) desejado. Scorsolini-Comin e Santos (2008) também apontaram, em sua revisão, que os pretendentes nacionais, ao traçarem o perfil da criança "ideal" do futuro filho(a), almejam como características a pouca idade, pele branca, ausência de doenças infectocontagiosas ou de anomalias congênitas, ou seja, um perfil seletivo que não retrata a realidade das crianças e adolescentes que estão disponíveis para adoção, como evidenciado constantemente no Cadastro Nacional de Adoção.

Pelos dados apresentados, pode-se considerar que a maioria das crianças/adolescentes brasileiros disponíveis para a adoção são de cor parda, do sexo masculino, maiores de dois anos e com irmãos. A partir desse panorama, observa-se que o perfil das crianças que estão disponíveis para adoção destoa daquele desejado pela maioria dos pretendentes. Isso faz com que a rigidez do perfil seja um aspecto negativo para o direito a convivência familiar, dificultando a inserção das crianças e dos adolescentes em um lar, bem como a demora para a satisfação do desejo de parentalidade (Pereira \& Azambuja, 2015; Rosa, Boris, Melo, \& Santos, 2016). Diante deste contexto, o presente estudo teve por objetivo apresentar uma revisão integrativa da literatura científica nacional e internacional acerca das adoções necessárias e de como esse tema vem sendo investigado e discutido no campo da adoção.

\section{Método}

\section{Tipo de estudo e pergunta norteadora}

Trata-se de um estudo de revisão integrativa da literatura científica que tem por objetivo apresentar uma síntese de vários estudos publicados e evidenciar conclusões gerais sobre uma específica área de estudo, com foco na produção de evidências para a prática (Mendes, Silveira, \& Galvão, 2008). A questão norteadora do presente estudo foi formulada a partir da estratégia 
PICO (Santos, Pereira, \& Nobre, 2007), empregada como metodologia em estudos de revisão voltados para a prática em evidências: "Quais tem sido os estudos na área da adoção necessária $(\mathrm{O})$ realizados no contexto nacional e internacional (I) na perspectiva das crianças, adolescentes, pais e profissionais (P)"? Como o objetivo não envolveu a comparação de cenários, a letra "C" não foi operacionalizada na redação da pergunta.

\section{Bases de dados e unitermos consultados}

As buscas foram realizadas nas bases eletrônicas/ bibliotecas LILACS, SciELO, PePISC e PsycINFO. Foram utilizadas combinações que abarcassem o tema dessa revisão: adoção necessária. Desta forma, utilizou-se o descritor adoção e as palavras-chaves adoção tardia, adoção inter-racial e adoção necessária. As combinações dos unitermos utilizados foram: adoção and doença crônica, adoção and deficiência, adoção and irmãos, adoção and HIV, adoção de crianças maiores e adolescentes. A pesquisa também foi realizada com as combinações em inglês e espanhol.

\section{Critérios de inclusão e de exclusão}

Para serem selecionados, os registros deveriam atender aos seguintes critérios pré-estabelecidos: (a) artigos científicos; (b) publicados em periódicos científicos, por se tratarem de estudos que passam por processo de avaliação e revisão de pares, ampliando as possibilidades de um rigoroso controle de qualidade acerca da avaliação desses materiais; (c) publicados em português, inglês e espanhol; (d) publicados entre janeiro de 2009 e dezembro de 2018, com a finalidade de abarcar o período de 10 anos; (e) disponíveis na íntegra; (f) que abarcassem a temática da adoção necessária, fornecendo indícios ou evidências para responder à pergunta norteadora.

Foram excluídos: (a) textos que não fossem artigos científicos, como teses, dissertações, monografias, livros, capítulos, resumos, obituários, resenhas, cartas, anais de congressos, editoriais; (b) materiais publicados antes do ano de 2009; (c) publicações distantes do tema investigado ou estudos que traziam tangencialmente a questão das adoções necessárias, apenas mencionando essa modalidade e não respondendo à pergunta norteadora; (d) artigos de revisão de literatura. Não houve seleção em relação a perspectivas ou abordagens teóricas.

\section{Procedimento}

A presente revisão adotou os procedimentos propostos por Mendes, Silveira e Galvão (2008), seguindo as fases: (1) levantamento bibliográfico nas bases de dados eletrônicas, realizada em dois dias no primeiro semestre de 2019; (2) leitura e análise dos materiais encontrados pelos títulos e resumos; (3) aplicação dos critérios de inclusão e exclusão; (4) seleção dos artigos a partir dos textos completos; (5) exclusão dos artigos repetidos; (6) composição do corpus a partir dos artigos recuperados e condizentes com os critérios de inclusão e exclusão; (7) composição do banco de dados em Excel, caracterizando as principais informações de cada estudo: título, autores, instituição de origem dos autores, ano de publicação, periódico, objetivo, tipo de estudo, amostra, instrumentos, principais resultados, principais conclusões e limites e potencialidades/contribuições e lacunas para novos estudos. Os passos de 1 a 6 foram realizados por dois juízes independentes, ambos pesquisadores da área de adoção e com experiência em estudos de revisão. As discordâncias foram avaliadas por um terceiro juiz e após esse processo foi composto o corpus analítico. A apresentação da revisão/ síntese do conhecimento pautou-se nas recomendações do sistema PRISMA a partir dos critérios preconizados em sua lista de verificação. Sendo assim, os artigos que compõem o corpus final foram analisados a fim de responder à questão norteadora. $\mathrm{O}$ corpus foi lido e analisado na íntegra e, a partir dos conteúdos abordados em cada produção e tentando responder à pergunta norteadora, foram construídas as seguintes categorias a posteriori: (a) Perfil dos estudos recuperados; (b) Preparação para a adoção: mudanças de paradigmas; (c) Adoção de crianças maiores: as dificuldades e os aspectos positivos dessa filiação; (d) Adoção de crianças com deficiência e/ou doença crônica: uma adoção possível?.

\section{Resultados e Discussão}

As buscas realizadas nas bases de dados empregando os unitermos selecionados resultaram em 12.414 registros. Os processos de busca, seleção e recuperação dos artigos estão ilustrados no fluxograma (Figura 1). A partir dos critérios de inclusão e exclusão e retirando os artigos que se repetiram, foram recuperados e analisados 35 artigos na íntegra, que compuseram o corpus da presente revisão.

\section{Perfil dos estudos recuperados}

Em relação às instituições de origem dos autores, as universidades que se destacaram foram: Universidade Federal do Pará e a Pontifícia Universidade Católica do Rio de Janeiro ( $\mathrm{n}=3 ; 8,57 \%)$. $\mathrm{O}$ ano de maior destaque 
foi o de 2014 ( $\mathrm{n}=9 ; 25,70 \%$ ). Os anos de 2010 e 2012 não apresentaram publicações nessa temática.

Os periódicos de maior evidência foram a Revista Estudos de Psicologia $(2013$; 2015; 2017) e a Revista da SPAGESP - Revista da Sociedade de Psicoterapia Analíticas Grupais do Estado de São Paulo (2014; 2015; 2016), Temas em Psicologia (2013; 2015; 2018), Contextos Clínicos $(2011 ; 2014 ; 2016)$ e Paidéia $(2009 ; 2011 ; 2016)$. A maioria das publicações ocorreu em periódicos nacionais $(\mathrm{n}=33 ; 94,3 \%)$, sendo apenas dois internacionais, Cuestiones de Infancia (2014) e American Journal Ortopsiquiatria (2014), com um artigo recuperado em cada. A maioria das publicações internacionais foi excluída por tratar de adoções transraciais, sendo estas não classificadas como adoções necessárias.

Quanto ao tipo de estudo, as publicações foram, predominantemente, com o enfoque empírico $(n=32$;
91,42\%), com destaque para a metodologia qualitativa $(\mathrm{n}=30 ; 85,71 \%)$. Dentre esses artigos, eles se classificavam em: estudo de caso ( $\mathrm{n}=7 ; 23.33 \%)$, exploratório ( $n=12 ; 40 \%)$, teórico-clínico $(n=4 ; 13,33 \%)$; estudo de caso múltiplo ( $\mathrm{n}=4 ; 13,33 \%)$, documental $(\mathrm{n}=2$; $6,67 \%$ ), e um estudo não especificou o tipo de enfoque utilizado. Um artigo se apresentou como estudo misto (quanti-quali) e apenas um com a abordagem quantitativa, descrevendo-se como um estudo documental, com caráter descritivo. Três artigos se caracterizaram como estudo teórico.

Em relação às amostras dos estudos, foi possível identificar alguns grupos. Os participantes que se destacaram nas publicações foram as famílias por adoção compostas por casais heterossexuais e os filhos por adoção (Fônseca, Santos, \& Dias, 2009; Levy, Diuana \& Pinho, 2009; Dugnani \& Marques, 2011; Otuka, Scorsoli-Comin, \& Santos, 2013; Merçon-Vargas, Rosa, \&

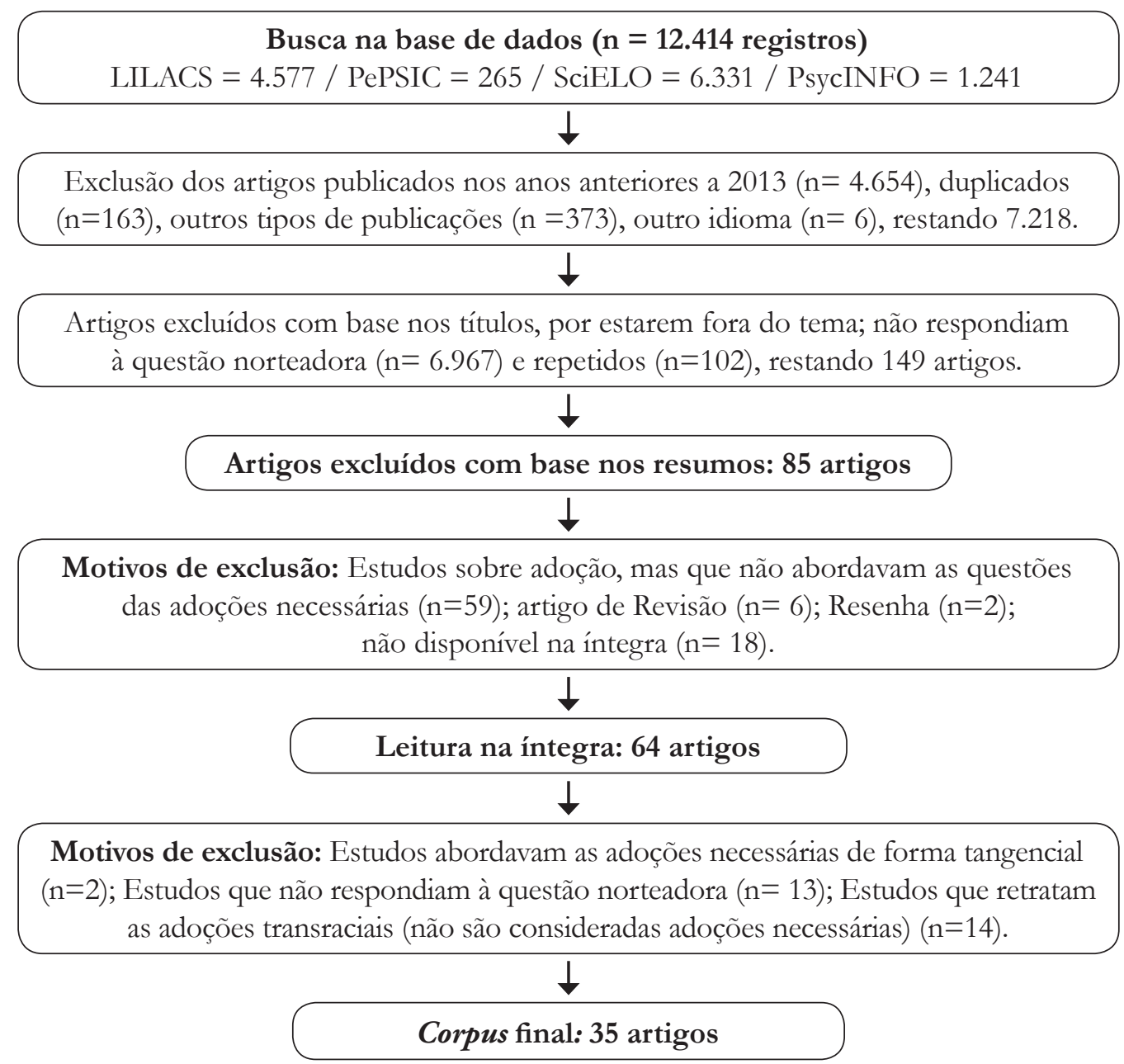

Figura 1. Fluxograma de busca, seleção e composição do corpus 
Dell’Aglio, 2014; Bicca \& Grzylowski, 2014; Sequeira \& Stella, 2014; Silva \& Benetti, 2015; Silva \& Cavalcante, 2015; Verceze, Silva, Oliveira, \& Sei, 2015; Mozzi \& Nuemberg, 2016; Andrade, Hueb, \& Alves, 2017; Combier \& Binkowski, 2017; Paulina, Ferreira, Bobato, \& Becker, 2018; Sampaio, Magalhães, \& Féres-Carneiro, 2018), seguidos por estudos com casais do mesmo sexo e filho por adoção (Dantas \& Ferreira, 2015; Machin, 2016; Coitinho, 2017), crianças que foram adotadas (Alvarenga \& Bittencourt, 2013; Vieira \& Henriques, 2013; Vieira, Dotta, Farias, \& Henriques, 2014; Gomes \& Levy, 2016), profissionais do Sistema Judiciário (Oliveira \& Pereira, 2011; Contente, Cavalcante \& Silva, 2013; Queiroz \& Brito, 2013), pessoas solteiras (Santos, Fônseca, Fônseca, \& Dias, 2011) e documentos, sendo esses um estudo sobre o Estatuto da Criança e do Adolescente, o Plano Nacional de Convivência Familiar e Comunitária e a Lei no 12.010/2009 (Machado, Ferreira, \& Seron, 2015), registro de pretendentes que constam no Cadastro Nacional de Adoção (Bossa \& Neves, 2018; Silva, Cavalcante, \& Dell'Aglio, 2016). E outro com os autos do processo judicial (Speck, Queiroz, \& Martin-Mattera, 2018).

Os instrumentos utilizados nas publicações foram diversificados. Nos estudos qualitativos foram utilizados: entrevista semiestruturada (Fônseca et al., 2009; Levy, Diuana, \& Pinho, 2009; Oliveira \& Pereira, 2001; Santos et al., 2011; Queiroz \& Brito, 2013; Otuka et al., 2013; Merçon-Vargas et al., 2014; Dantas \& Ferreira,
2015; Silva \& Benetti, 2015; Gomes \& Levy, 2016; Mozzi \& Nuemberg, 2016; Andrade, Hueb, \& Alves, 2017; Paulina et al., 2018; Sampaio et al., 2018), diário de campo (Merçon-Vargas et al., 2014; Bicca \& Grzylowski, 2014; Silva \& Benetti, 2015; Combier \& Binkowski, 2017), narrativa de vida (Vieira \& Henriques, 2013; Vieira et al., 2014), questionário sociodemográfico (Otuka et al., 2013; Mozzi \& Nuemberg, 2016), observação direta (Queiroz \& Brito, 2013; Merçon-Vargas et al., 2014); documentos (Machado et al., 2015; Speck et al., 2018), questionário (Dugnani \& Marques, 2011; Contente, Cavalcante, \& Silva, 2013), entrevista em profundidade (Machin, 2016), entrevista com pergunta disparadora (Silva \& Santos, 2014), procedimento desenho-estória com tema (Andrade et al., 2017) e narração de histórias infantis (Andrade et al., 2017). O estudo com o enfoque quanti-qualitativo (Silva \& Cavalcante, 2015) utilizou entrevista semiestruturada, inventário de rotina e diário de campo para a coleta dos dados. Já o estudo com abordagem quantitativa (Silva et al., 2016) utilizou os registros do Cadastro Nacional da Adoção. Três estudos não especificaram o que utilizaram como instrumento para a coleta de dados.

Os objetivos dos estudos recuperados foram variados, apresentando quatro principais eixos (Tabela 1), com destaque para a amostra e o tipo de adoção necessária. $\mathrm{O}$ tipo de adoção necessária abordada nos estudos foi restrito, sendo que na maior parte se discutiam questões da adoção de crianças maiores, seguidas

Tabela 1

Principais objetivos encontrados nos estudos recuperados

Principais objetivos dos estudos recuperados na revisão

Exemplos de estudos

Refletir/Analisar a preparação de crianças e pretendentes à adoção

Descrever, compreender a adoção de crianças com deficiência/doença crônica Analisar, discutir, conhecer a adoção de crianças maiores

Compreender, apresentar a adoção de irmãos
Levy et al. (2009); Oliveira \& Pereira, 2011; Contente et al. (2013); Gomes \& Levy (2016); Sequeira \& Stella (2014); Coitinho (2017); Machin (2016).

Fônseca et al. (2009); Silva \& Cavalcante (2015); Mozzi \& Nuemberg (2016); Silva et al. (2016); Boberiene \& Yazykova (2014); Rodrigues \& Hennigen (2014); Bossa \& Neves (2018)

Dugnani \& Marques (2011); Santos et al. (2011); Alvarenga \& Bittencourt (2013); Queiroz \& Brito (2013); Vieira \& Henriques (2013); Vieira, Dotta, Farias, \& Henriques (2014); Merçon-Vargas et al. (2014); Otuka et al. (2013); Bicca \& Grzybowski (2014); Salmun (2014); Dantas \& Ferreira (2015); Machado et al. (2015); Silva \& Benetti (2015); Verceze et al. (2015); Andrade et al. (2017); Combier \& Binkowski (2017); Paulina et al. (2018); Sampaio et al. (2018); Speck et al. (2018)

Couto (2014); Silva \& Santos (2014) 
pela adoção de crianças com deficiência física e doença crônica e, por último, a adoção de irmãos. Os estudos que retratavam a adoção de irmãos estavam relacionados à adoção de crianças maiores, já que os participantes foram adotados com mais de dois anos de idade.

\section{Preparação para a adoção: mudanças de paradigmas}

Como vimos, com o ECA a adoção passou a ser uma possibilidade de família, e a partir dele novas leis foram criadas para garantir de fato esses direitos. A Nova Lei da Adoção contribuiu com algumas alterações no ECA para que o bem-estar da criança viesse a ser o objetivo central das problematizações. Atualmente, a Lei $\mathrm{n}^{\circ}$ 13.509, de 22 de novembro de 2017, dispõe de algumas mudanças, principalmente em relação a prazos, para visibilizar o processo.

Estudos ressaltam a importância da Lei $\mathrm{n}^{\circ}$ 12.010/2009 por estabelecer a necessidade de preparação tanto dos postulantes como também das crianças e adolescentes para a adoção (Contente et al., 2013; Machado et al., 2015; Merçon-Vargas et al., 2014; Oliveira \& Pereira, 2011). No entanto, essa preparação não é uniforme em todo o país, pois na legislação só é indicado que esse trabalho seja realizado conforme a demanda da Comarca e da população local (Machado et al., 2015). Contente et al. (2013) e Merçon-Vargas et al. (2014) apontam essa falta de padronização dessas orientações em conformidade com a Nova Lei da Adoção, fazendo com que na prática isto ainda não tenha sido alcançado com total êxito, já que as orientações propostas não vão ao encontro da real necessidade dos postulantes e das crianças para a adoção.

As pesquisas apontaram que essa preparação deve ser realizada com os profissionais do Juizado e da instituição de forma conjunta, com o objetivo de orientar tanto as crianças como os pretendentes. A preparação dos pretendentes envolve tanto a parte do Judiciário como também a participação nos grupos de apoio psicossocial. Esses grupos têm por objetivo principal sensibilizar os pais em relação às diversas questões presentes na filiação por adoção, é um espaço que procura refletir sobre a decisão de adotar, as motivações, os medos e fantasias inerentes do processo, bem como a construção da parentalidade. Também atua no processo de reflexão sobre a escolha de adotar e a desidealização da família. De acordo com Levy et al. (2009) e Machado et al. (2015), a Nova Lei da Adoção reitera a necessidade de preparação dos postulantes quanto ao perfil das crianças que vivem na instituição para a adoção, com destaque para aqueles que são preteridos pelos adotantes, ou seja, os grupos de irmãos, as crianças maiores e adolescentes, com deficiência ou adoecimento e as adoções interraciais. Essa mudança de perfil é essencial, já que o filho imaginado é incompatível com o perfil das crianças para a adoção. Existe ainda a procura por bebês com até um ano, brancos e do sexo feminino.

Silva et al. (2016) destacaram que o perfil dos pretendentes está em transformação. Em seu estudo os pretendentes adotaram crianças com adoecimento e/ou doença crônica, não demonstrando exigências quanto a condição de saúde, cor ou sexo, o que pode apontar para uma maior flexibilidade quanto a "crianças vulneráveis" (termo adotado pelos autores do artigo).

Rodrigues e Hennigen (2014) discutem o Projeto de Lei (Almeida, 2011) que tramitou na Câmara dos Deputados e que previa o dobro da dedução no imposto de renda para aqueles pretendentes que adotassem crianças com adoecimento grave ou deficiência, afrodescendentes e aqueles que tivessem com mais de dois anos de idade. A reflexão se pautou no paradoxo solidariedade versus relação de mercado, o que nos faz pensar qual o objetivo central da Adoção nessa prática, o melhor interesse da criança ou a relação custo/ benefício para os pretendentes? Esse incentivo financeiro também foi apontado por Oliveira e Pereira (2011), em que esse tipo de relação é passível de questionamentos, como: até que ponto é o real desejo pela filiação por adoção? A família, segundo esses autores, acaba se fundamentando em relações econômicas e não no bem-estar da criança e do adolescente como é previsto pelo ECA (1999).

O que é apontado na Nova Lei da Adoção e vem sendo reforçado pela Lei $\mathrm{n}^{\circ} 13.509$, de 22 de novembro de 2017, a necessidade de se pensar as verdadeiras crianças que estão nas instituições para adoção e com isso repensar o desejo da parentalidade. Como é discutido por Levy et al. (2009) e Stella e Sequeira (2014), os pretendentes devem analisar a real disponibilidade para se ter um filho e o que isso significa, independentemente de qualquer característica atribuída a essa criança. $\mathrm{O}$ filho consanguíneo também pode apresentar problemas a qualquer instante, de modo que o filho perfeito existe apenas no imaginário. A criança real é constituída por diversas características, como a personalidade, questões físicas ou emocionais, isso independentemente de qualquer tipo de filiação.

Coitinho (2017), Machin (2016), Merçon-Vargas et al. (2014) e Rodrigues e Hennigen (2014) apontam que as adoções necessárias são realizadas em maior 
parte pelos pretendentes de outros países (adoções internacionais) e por casais do mesmo sexo. Os estudos identificaram que esses pretendentes fazem com que o direito à convivência familiar e comunitária seja proporcionado a essas "crianças vulneráveis", justamente por essas pessoas buscarem um perfil que destoa daquele que é mais concorrido, ampliando as chances em relação ao padrão de adoção que acontece no Brasil. Há que se ressaltar que a adoção internacional é apontada como a última alternativa para a adoção, priorizando-se os pretendentes residentes no Brasil. Desta forma, mostra-se a importância da preparação dos pretendentes quanto ao perfil das crianças, possibilitando o amadurecimento do projeto parental, o que também pode envolver uma reflexão sobre o real desejo pela filiação, a satisfação de motivações inconscientes, como por exemplo, o luto, a infertilidade, a manutenção da conjugalidade, entre outros. Esse processo de amadurecimento tem sido fomentado nos cursos preparatórios com todos os postulantes, independentemente de suas características psicossociais e configurações familiares.

De acordo com Machin (2016), o perfil dos casais heterossexuais se destaca pela preferência de crianças com idade inferior a um ano, menina, com o fenótipo similar aos pais adotivos. Esse perfil ideal representa a forma como essa criança se inscreve no núcleo familiar, a partir de princípios relativos ao modelo biológico/consanguíneo de reprodução. Esse processo fomenta vários temas essenciais para trabalhar com essas famílias. O perfil do filho está relacionado à aceitação social? O tempo de convivência com a família consanguínea tem influência sobre essa decisão? Atualmente, como é apontado pela literatura (Levy et al., 2009; Merçon-Vargas et al., 2014; Sequeira \& Stella, 2014), o trabalho realizado com os pretendentes é de conscientização, em que a prioridade não são os interesses dos futuros pais, mas sim o da criança. A principal premissa é dar uma família à criança, não mais dar um filho para os que não podem tê-lo, o que permite abarcar o desejo de casais do mesmo sexo, pessoas solteiras, entre outros postulantes, como legítimo, independentemente de suas características.

Ao elencar e fomentar nos cursos preparatórios que qualquer pretendente habilitado para a adoção pode pleitear ou assumir uma adoção considerada necessária, opera-se um movimento que não torna esse tipo de adoção vinculado exclusivamente a pretendentes "mais flexíveis", "menos exigentes" ou que sejam oriundos de "configurações consideradas menos tradicionais" (como pessoas solteiras ou casais do mesmo sexo, por exemplo), mas justamente conscientiza o grupo de postulantes acerca da necessidade do amadurecimento parental, processo este que deve transpor fantasias e desejos individuais e normativos em prol do melhor interesse da criança/adolescente.

\section{Adoção de crianças maiores: dificuldades e os aspectos positivos dessa filiação}

A adoção de crianças maiores, antigamente conhecida como adoção tardia, refere-se às adoções de crianças com idade superior a dois anos e os adolescentes. A literatura aponta alguns fatores que favorecem a esse tipo de adoção, como o papel da preparação dos postulantes, em que após essa etapa os futuros pais repensam o perfil do filho que desejam ter e, com isso, se tornam mais abertos a modificar as características da criança que almejavam adotar (Bicca \& Grzybowski, 2014; Levy et al., 2009; Queiroz \& Brito, 2013).

Esses pretendentes passam a ter uma maior consciência do processo de adoção e com isso as dificuldades que podem ser apresentadas na adoção de uma criança maior passam a ser insignificantes em relação à motivação para adotá-la. Queiroz e Brito (2013) ressaltam que os problemas podem ser superados a partir da vinculação entre pais e filhos, a construção do amor e da dedicação dispensados a essa relação. Santos et al. (2001) e Bicca e Grzybowski (2014) apontaram como aspectos facilitadores entre a adoção de crianças maiores e os postulantes a adoção: a idade, conhecimento da sua própria história de vida, o desejo de exercer a paternidade e a maternidade.

A criança, com o passar do tempo, passa a ter uma maior autonomia e com isso menos dependência dos pais, conseguindo acompanhá-los em suas atividades e exigindo menos cuidados básicos no dia-a-dia. Ao conhecer sua própria história de vida possibilita-se o conhecimento acerca do seu processo de adoção, o rompimento com a família de origem e, com isso, maior abertura para uma relação mais franca e verdadeira, com menos fantasias relacionadas a esse aspecto (Dugnani \& Marques, 2011). O real desejo de vivenciar a paternidade e a maternidade faz com que os pretendentes sejam indiferentes às características específicas das crianças, sendo abertos a acolher independentemente da idade, cor, sexo e a presença de adoecimento. Desta forma, esses fatores fazem com que a adoção das crianças e dos adolescentes seja satisfatória (Bicca \& Grzybowski, 2014; Dantas \& Ferreira, 2015).

No entanto, a maioria dos estudos recuperados apontaram as dificuldades que tanto os pretendentes 
quanto as crianças e os adolescentes podem vivenciar nesse processo de adoção. As crianças que são caracterizadas por esse tipo de adoção são marcadas por histórias significativas, são sobreviventes de várias rejeições, inseguranças, abandonos, maus-tratos, abusos, devoluções, entre outros (Alvarenga \& Bittencourt, 2013; Couto, 2014; Silva \& Benetti, 2015; Silva \& Santos, 2014; Vieira et al., 2014; Paulina et al., 2018; Sampaio et al., 2018, Speck et al., 2018). O período de transição dessa nova família é caracterizado por importantes modificações no estilo de vida após a chegada da criança. Essas mudanças são comuns e necessárias, independentemente do tipo de filiação, promovendo o surgimento de novos papéis. Nesse processo de transição, a família composta por adoção de crianças maiores pode apresentar algumas dificuldades em decorrência da adaptação da criança e dos pais, como dificuldades no aprendizado escolar, em aceitar os hábitos da nova família, atitudes de desafio em relação a regras e limites estabelecidos, manifestações de raiva, problemas de saúde, não aceitação pelos familiares e preconceito social (Santos el., 2011; Bicca \& Grzybowski, 2014; Otuka, Scorsolini-Comin, \& Santos, 2013; Speck et al., 2018; Paulina et al., 2018). Essas características, no entanto, não podem ser tomadas como deterministas e exclusivamente presentes em adoções e, no caso, de adoções necessárias, de modo que é preciso retomar que o processo de desenvolvimento é contínuo, dinâmico e que atravessamentos, rupturas e declínios também compõem o repertório ao longo do ciclo vital quaisquer que sejam os tipos de filiação e configurações familiares (Santos et al., 2011).

Dugnani e Marques (2011), Couto (2014), Combier e Binkowski (2017), Vieira et al. (2014) abordam a necessidade dessas crianças significarem sua história de vida, já que a má elaboração pode acarretar consequências negativas que se refletem em outros meios de atuação, como relacionamentos, escola, socialização e profissão. A família é o meio que pode dar viabilidade para a criança reviver as experiências angustiantes e com isso possibilitar com que ela se sinta pertencente ao ambiente que a cerca (Alvarenga \& Bittencourt, 2013; Andrade et al., 2017).

Dugnani e Marques (2011) e Verceze et al. (2015) apontam que a não aceitação do passado do filho(a) faz com que a criança não se sinta integrada à família, gerando na mesma dificuldade de se colocar dentro do contexto familiar. Tais pesquisas também apontam a atitude das famílias por adoção ao atribuir os comportamentos inadequados da criança à herança da família de origem. De acordo com Couto (2014), a criança precisa vivenciar o luto da família de origem e elaborar a sua história para poder confiar em outras pessoas com o passar do tempo. A chamada nova família deve ser aberta à escuta e acolher as necessidades dessas crianças, respeitar a história de vida e suas vivências e ajudá-las a reintegrar a história anterior com a nova história que está por vir, contribuindo para a criação do vínculo paterno-filial e a construção de uma relação segura (Combier \& Binkowski, 2017; Sampaio et al., 2018; Speck et al., 2018).

O período inicial pode ser marcado por vários acontecimentos, em decorrência da adaptação de todos os integrantes da família. De acordo com Combier e Binkowski (2017), Otuka et al. (2013) e Verceze et al. (2015), tanto os pais por adoção como os consanguíneos podem projetar no filho expectativas que, muitas vezes, podem não ser correspondidas. Otuka et al. (2013) ressaltam que muitas vezes os pais atribuem um papel idealizado para o filho por adoção, caracterizando-o como o filho perfeito e, com isso, impossibilitam que o mesmo possa exprimir sentimentos hostis e agressivos para não decepcionar a fantasia dos pais. Desta forma, a criança passa a desenvolver comportamentos que são esperados pelos pais, preocupa-se em se mostrar uma pessoa agradável e adequada à nova demanda da família, teme a fragilidade do vínculo com a nova relação e, como consequência dos seus maus comportamentos, uma nova situação de abandono (Verceze et al., 2015; Sampaio et al., 2018). A criança passa a não expressar seus próprios sentimentos, comportando-se de forma passiva em relação ao meio.

Outro comportamento característico é a testagem, a criança que foi adotada pode testar o amor dos pais para ver até que ponto eles são capazes de suportar e não vão devolvê-la, ou seja, eles procuram verificar se realmente não vão ser abandonados e devolvidos novamente. O pais por adoção devem saber suportar e não desistir da criança, de modo que, ao sobreviver às manifestações de sentimentos negativos, podem construir uma relação mais segura com o filho (Gomes \& Levy, 2016; Silva \& Benetti, 2015; Verceze, Silva, Oliveira, \& Sei, 2015; Sampaio et al., 2018; Speck et al., 2018).

O fortalecimento desse vínculo também pode ser influenciado pelas relações que se estabelecem com o meio externo. A rede de apoio social foi citada pelas pesquisas como principal recurso dessas famílias para enfrentar as dificuldades. Os estudos citaram os familiares, amigos, vizinhos, grupo de apoio a adoção, professores da criança e a equipe técnica do Fórum 
como integrantes da rede de apoio que os auxiliam para superar os desafios (Santos et al., 2011; Bicca \& Grzybowski, 2014; Queiroz \& Brito, 2013; Paulina et al., 2018; Silva \& Santos, 2014; Speck et al., 2018). É importante destacar que a vinculação na filiação por adoção é gradativa. De acordo com Paulina et al. (2018), o histórico da adoção tem suas peculiaridades, no entanto, a vinculação é construída, o contato diário faz com que consolide a afetividade entre os envolvidos. O que também podemos observar na parentalidade consanguínea.

Vieira et al. (2014) destacaram a escola como um ambiente externo à família que pode favorecer a construção da identidade da criança que foi adotada, sendo um lugar de mudança de valores pessoais e de referência ao longo do desenvolvimento psicossocial do sujeito. A escola pode ser considerada o segundo lugar de relação entre pares depois da família, de modo que esse ambiente pode desempenhar um papel essencial na organização da identidade e da personalidade.

O acompanhamento psicológico foi apontado nos estudos (Santos et al., 2011; Silva \& Benetti, 2015; Gomes \& Levy, 2016; Alvarenga \& Bittencourt, 2013; Verceze et al., 2015) como um dos recursos de enfrentamento e potencialização tanto dos pais como das crianças para lidarem com as mudanças da nova família. Para a criança, a psicoterapia é apontada como um espaço de externalização e elaboração de angústias vivenciadas no processo e também de fortalecimento de vínculo (Santos et al., 2011; Gomes \& Levy, 2016). Para os pais, esse acompanhamento faz com que eles expressem as suas fantasias e sentimentos sobre a criança, sendo uma oportunidade para pensar em transformações (Silva \& Benetti, 2015; Speck et al., 2018). Também é uma forma de preparação desses pais para que possam promover o acolhimento dos seus futuros filhos, bem como estimular a criação de vínculos de confiança, fazendo com que suportem os ataques e confrontações da criança independentemente de qualquer situação (Alvarenga \& Bittencourt, 2013).

\section{Adoção de crianças com deficiência e/ ou doença crônica: uma adoção possivel?}

A adoção de crianças com deficiência e ou doença crônica não foi muito abordada pelos estudos recuperados, sendo que apenas seis pesquisas retrataram essa temática, cinco no Brasil (Fônseca et al., 2009; Silva \& Cavalcante, 2015; Mozzi \& Nuernberg, 2016; Silva, Cavalcante, \& Dell'Aglio, 2016; Bossa \& Neves, 2018) e uma na Rússia (Boberiene \& Yazykova, 2014). Esse tipo de adoção demonstrou semelhanças e diferenças quanto ao contexto de cada país. A principal semelhança foi em relação à institucionalização, a realidade dessas pessoas que são direcionadas ao acolhimento, ao qual as famílias de origem não puderam oferecer o cuidado necessário para seus filhos, com destaque para os indivíduos que precisam de um olhar mais delicado em decorrência do adoecimento. As crianças com adoecimento, tanto no Brasil como na Rússia, quando chegam nas instituições de acolhimento precisam de cuidados específicos. No entanto, nem sempre o local apresenta os recursos necessários, de modo que esse direito permanece negligenciado (Boberiene \& Yazykova, 2014; Bossa \& Neves, 2018; Rossetti-Ferreira et al., 2012; Silva et al., 2016).

As diferenças em relação à promoção dessas adoções entre esses dois países são as políticas públicas que priorizam o bem-estar e a convivência familiar e comunitária dessas crianças e adolescentes. De acordo com Boberiene e Yazykova (2014), a política de adoção da Rússia restringe os pretendentes de alguns países, como Estados Unidos, Canadá, Nova Zelândia e França, por exemplo. Já no Brasil algumas leis foram criadas dando prioridade para essas adoções. A Lei $\mathrm{n}^{\circ} 12.955 / 2014$ propôs alteração no parágrafo $9^{\circ}$ do artigo 47 do ECA, em que estabelece prioridade nos processos de adoção quando o adotando for uma criança com necessidades especiais, ou com algum quadro de adoecimento crônico (Brasil, 2014). Bossa e Neves (2018) apontam que em 2017, ao consultar o Cadastro Nacional de Adoção, foi possível perceber que a maioria dos postulantes à adoção não aceitam crianças e adolescentes com doença crônica ou deficiência. Dentre os pretendentes que aceitam, há maior abertura para a deficiência física em relação aos transtornos mentais.

De acordo com Silva et al. (2016), a partir dos dados apresentados pelo Cadastro Nacional de Adoção de 2008 a 2012, foi possível verificar que o perfil de destaque dos pretendentes que optam por esse tipo de adoção foi: estado civil casado, cor de pele branca, proveniente de família de baixo e médio nível socioeconômico, não possui filhos (tanto consanguíneo como por adoção) e escolaridade de nível médio completo. Esses postulantes à adoção não demonstraram exigências quanto à cor e ao sexo do futuro filho.

A literatura sugere que esses futuros pais inicialmente não decidiram pela adoção de crianças e adolescentes com adoecimento. A motivação inicial desses pais não se difere dos outros tipos de adoção, no entanto, o que pode influenciar na decisão final é a preparação psicológica, os recursos financeiros da família e 
também ao acesso à informação. A motivação foi sendo construída, uma vez que com a preparação sabiam qual era a realidade das crianças presentes nas instituições de acolhimento. Esses estudos também apontaram como fator que favorece a adoção dessas crianças a experiência anterior da família com a condição da enfermidade ou da cronicidade (Fônseca et al., 2009; Mozzi \& Nuernberg, 2016; Silva \& Cavalcante, 2015).

O recurso financeiro e a rotina da família foram apontados pelos estudos recuperados como fatores determinantes para esse tipo de adoção (Bossa \& Neves, 2018; Mozzi \& Nuernberg, 2016; Silva \& Cavalcante, 2015). Normalmente, dependendo do tipo de adoecimento, a rotina é vivida a partir das necessidades da criança devido aos acompanhamentos com diferentes profissionais, como médicos, fisioterapeutas, fonoaudiólogos, entre outros. A dinâmica e a rotina da família são centralizadas para prover o melhor cuidado da criança e, com isso, o meio precisa se reorganizar estruturalmente e também no aspecto financeiro, já que as despesas são direcionadas para a promoção de saúde do adotando (Bossa \& Neves, 2018; Fônseca et al., 2009; Silva \& Cavalcante, 2015).

As especificidades no cuidado fazem com que a família se reinvente, alguém passa a ser o cuidador direto das necessidades básicas da criança e, com isso, a sua vida passa a ter novos sentidos, como a falta de disponibilidade de tempo e novas responsabilidades. A rede de apoio foi citada como um recurso para amenizar e enfrentar as dificuldades, de modo que a aceitação e o apoio são essenciais para essa nova configuração familiar. A forma como a família se relaciona com o ambiente externo pode interferir também no desenvolvimento e na adaptação da criança (Fônseca et al., 2009; Mozzi \& Nuernberg, 2016; Silva \& Cavalcante, 2015). A escola, a família extensa e os cuidados na rotina são aspectos essenciais para a promover a interdependência e a qualidade de vida não só da criança com o adoecimento, mas também do núcleo familiar (Fônseca et al., 2009; Mozzi \& Nuernberg, 2016; Silva \& Cavalcante, 2015).

\section{Considerações Finais}

O presente estudo permitiu compreender como as adoções necessárias estão sendo abordadas na literatura científica. No entanto, ficou evidente a existência de poucas publicações internacionais. Uma das possibilidades é que o conceito de adoções necessárias seja uma definição brasileira e que deflagre uma demanda nacional e contextual. No Brasil, há leis que favorecem e promovem esses tipos de adoções por serem a maioria das crianças que estão disponíveis para a adoção nas instituições de acolhimento. Os artigos internacionais recuperados retrataram as adoções de crianças com deficiência ou adoecimento, com destaque para a realidade da Rússia e a de crianças maiores, com o enfoque nas adoções internacionais.

A maioria das publicações destacou a realidade brasileira e os tipos de adoções pesquisadas foram: adoção de crianças maiores e alguns estudos sobre adoção de crianças com deficiência e adoecimento. Apenas dois estudos retrataram a adoção de irmãos, mas o enfoque foi relacionado à adoção de crianças maiores. Desta forma, ficou evidente o maior aprofundamento nas questões que permeiam a realidade das adoções de crianças maiores, com o objetivo de dar visibilidade a esse tipo de adoção, demonstrando as principais características, principalmente em relação à adaptação e à história de vida da criança e/ou adolescente.

Os estudos relacionados à adoção de crianças com deficiência e adoecimento crônico retrataram a rotina dessas famílias, o direcionamento para o cuidado e também as estratégias adotadas, a possibilidade de se realizar essa adoção com o apoio e cuidado não só pela família nuclear, mas também pela rede de apoio que essa família tem acesso. Desta forma, de acordo com as evidências recuperadas, ainda há poucas pesquisas relacionadas à adoção de irmãos e nesse estudo proposto nenhuma pesquisa retratava as adoções inter-raciais, o que demonstra a necessidade de maior investigação nessa área.

Destaca-se também a necessidade de conscientização dos pretendentes quanto ao perfil das crianças para adoção nas instituições de acolhimento, o que pode ser potencializado tanto pelos grupos psicossociais como pelos profissionais da Vara da Infância e Juventude. Em suma, a revisão possibilitou conhecer as peculiaridades desses tipos de adoções, demonstrando o que tem sido produzido e a necessidade de reconhecer que se tratam de adoções possíveis e que, por isso mesmo, merecem maior destaque na produção científica da área.

\section{Referências}

Almeida,C.(2011).ProjetodeLein ${ }^{\circ}$ 942,de2011.Recuperado de http://www.camara.gov.br/proposicoesWeb/ fichadetramitacao?idProposicao $=497492$

Alvarenga, L. L., \& Bittencourt, M. I. G. F. (2013). A delicada construção de um vínculo de filiação: o 
papel do psicólogo no processo de adoção. Pensando Famílias, 17(1), 41-53.

Andrade, L. C. S., Hueb, M. F. D., \& Alves, C. M. P. (2017). Era uma vez... um estudo de caso sobre histórias e estórias adotivas. Estudos de Psicologia (Campinas), 34(1), 173- 183. https://doi. org/10.1590/1982-02752017000100017

Bicca, A., \& Grzybowski, L. S. (2014). Adoção tardia: percepções dos adotantes em relação aos períodos iniciais de adaptação. Contextos Clínicos, 7(2), 155- 167.

Boberiene, L. V., \& Yazykova, E. (2014). Children with disabilities in Russian institutions: Can the West help protect the most vulnerable? American Journal of Orthopsychiatry, 84(3), 266-272. https://doi. org/10.1037/h0099813

Bossa, D. F., \& Neves, A. S. (2018). O Unheimlich na adoção da criança com deficiência. Cadernos de Psicanálise, 40(8), 97-109.

Brasil (2017). Lei no 13.509, de 22 de novembro de 2017. Dispõe sobre adoção e altera a Lei $n^{\circ}$ 8.069, de 13 de julho de 1990 (Estatuto da Criança e do Adolescente). Recuperado de htttp:// http://www2.camara.leg. br/legin/fed/lei/2017/lei-13509-22-novembro-2017-785783-publicacaooriginal-154279-pl.html.

Brasil, Congresso Nacional, \& Câmara dos Deputados (2014). Lei $n^{\circ} 12.955$ de 2014. Recuperado de http:// www.planalto.gov.br/CCIVIL_03/_ Ato2011-2014/2014/Lei/L12955.htm

Brasil, Conselho Nacional de Justiça. (2013). Encontro e desencontros da adoção no Brasil: uma análise do Conselho Nacional de Justiça (CNJ). Recuperado de http:/ / www.cnj.jus.br/images/pesquisas-judiciarias/Publicacoes/pesq_adocao_brasil.pdf

Brasil (2009, 4 de agosto). Lei n. 12.010, de 3 de agosto de 2009. Dispõe sobre adoção. Brasília. Diário Oficial da União, Seção 1.

Brasil (1990). Lei no 8. 069, de 13 de julho de 1990. Dispõem sobre o Estatuto da Criança e do Adolescente e dá outras providências. Diário Oficial da União, 27 de setembro de 1990. Recuperado de http://www.planalto.gov.br/ccvil_03/leis/ L8069.htm

Combier, C. V., \& Binkowski, G. (2017). Adoção e mito: os destinos do "mito familiar" na cena da família contemporânea. Estudo a partir de um caso clínico de adoção na França atual. Ágora, 20(1), 159- 172. https://doi.org/10.1590/s1516-14982017001009

Contente, S. R., Cavalcante, L. I. C., \& Silva, S. S. C. (2013). Adoção e preparação infantil na percepção dos profissionais do Juizado de Infância e Juventude de Belém/PA. Temas em Psicologia, 21(2), 317-333. https://doi.org/10.9788/TP2013.2-02

Coitinho, R. A. F. (2017). Sob o "melhor interesse"! O "homoafetivo" e a criança nos processos de adoção. Estudos Feministas, 25(2), 495- 518. https://doi. org/10.1590/1806-9584.2017v25n2p495

Couto, O. H. C. (2014). Os caminhos cruzados da adoção: do abandono precoce à última esperança. Reverso, 36(68), 63-70.

Dantas, F. S. S., \& Ferreira, S. P. A. (2015). Adoção tardia: produção de sentidos acerca da paternagem e filiação em uma família homoafetiva. Temas em Psicologia (Ribeirão Preto), 23(3), 593-606. https://doi. org/10.9788/TP2015.3-06

Dugnani, K. C. B., \& Marques, S. L. (2011). Construção e validação de instrumento para prática interventiva na adoção. Paidéia, 21(50), 317-328. http:// dx.doi.org/10.1590/S0103-863X2011000300004

Ferreira, F. R. F. (2014). O preço da criança e a Nova Cultura da Adoção: do cenário político-legal às práticas de adoção em Natal/RN. Estudos de Sociologia, 19(36), 61-80.

Fônseca, C. M. S. M. S., Santos, C. P., \& Dias, C. M. S. B. (2009). A adoção de crianças com necessidades especiais na perspectiva dos pais adotivos. Paidéia, 19(44), 303-311. http://dx.doi.org/10.1590/ S0103-863X2009000300004.

Ghirardi, M. L. A. M. (2009). A devolução de crianças adotadas: rupturas do laço familiar. Pediatria Moderna, 45(2), 66-70.

Gomes, I. C., \& Levy, L. (2016). A Psicanálise vincular e a preparação das crianças para a adoção: uma proposta terapêutica e interdisciplinar. Contextos Clínicos, 9(1), 109-117.

Levy, L., Diuana, S., \& Pinho, P. G. R. (2009). O grupo de reflexão como estratégia de promoção de saúde com famílias adotivas. Mudanças-Psicologia da Saúde, 17(1), 39-42.

Machado, L. V., Ferreira, R. R., \& Seron, P. C. (2015). Adoção de crianças maiores: sobre aspectos legais 
e construção do vínculo afetivo. Estudos Interdisciplinares em Psicologia, 6(1), 65-81. https://doi. org/10.5433/2236-6407.2015v6n1p65

Machin, R. (2016). Homoparentalidade e adoção: (Re)afirmando seu lugar como família. Psicologia \& Sociedade, 28(2), 350-359. https://doi. org/10.1590/1807-03102016v28n2p350

Mendes, K. D. S., Silveira, R. C. C. P., \& Galvão, C. M. (2008). Revisão integrativa: método de pesquisa para a incorporação de evidências na saúde e na enfermagem. Texto Contexto Enfermagem, 17(4), 758-764. https://doi.org/10.1590/ S0104-07072008000400018

Merçon-Vargas, E. A., Rosa, E. M., \& Dell'Aglio, D. D. (2015). A produção científica e os aspectos legais no âmbito da adoção nacional e internacional. In F. Scorsolini-Comin, A. K. Pereira, \& M. L. T. Nunes (Orgs.), Adoção: legislação, cenários e práticas (pp. 2962). São Paulo: Vetor.

Merçon-Vargas, E. A., Rosa, E. M., \& Dell'Aglio, D. D. (2014). Adoção nacional e internacional: significados, motivações e processos de habilitação. Revista da SPAGESP, 15(2), 12-26.

Mozzi, G., \& Nuernberg, A. H. (2016). Adoption of children with disabilities: a study with adoptive parents. Paidéia, 26(63), 101-109. https://doi. org/10.1590/1982-43272663201612

Oliveira, C. A., \& Pereira, E. G. (2011). Estudo sobre a Lei Nacional de Adoção: institucionalização, acolhimento familiar e cadastros. Contextos Clínicos, 4(2), 113-122. doi:10.4013/ctc.2011.42.05

Otuka, L. K., Scorsolini-Comin, F., \& Santos, M. A. (2013). Adoção tardia por casal divorciado e com filhos biológicos: novos contextos para a parentalidade. Estudos de Psicologia, 30(1), 89-99. https://doi. org/10.1590/S0103-166X2013000100010

Paulina, E., Ferreira, L., Bobato, S. T., \& Becker, A. P. S. (2018). Processo de vinculação afetiva de crianças adotadas na perspectiva dos pais adotantes. Boletim Academia Paulista de Psicologia, 38(94), 77-94.

Pereira, A. H., \& Azambuja, M. R. F. (2015). História e legislação da adoção no Brasil. In F. Scorsolini-Comin, A. K. Pereira, \& M. L. T. Nunes (Orgs.), Adoção: legislação, cenários e práticas (pp. 17-27). São Paulo: Vetor.
Queiroz, A. C. A., \& Brito, L. (2013). Adoção tardia: o desafio da garantia do direito à convivência familiar e comunitária. Textos \& Contextos, 12(1), 55-67.

Rodrigues, L., \& Hennigen, I. (2014). Visibilidades da adoção: interrogando estratégias e práticas sociais. Psicologia Argumento, 32(76), 93- 103. https://doi. org/10.7213/psicol.argum.32.076.AO05

Rosa, J. M., Boris, G. D. J. B., Melo, A. K., \& Santos, M. A. (2016). A construção dos papéis parentais em casais homoafetivos adotantes. Psicologia: Ciência e Profissão, 36(1), 210-223. https://doi. org/10.1590/1982-3703001132014

Rossetti-Ferreira, M. C., Almeida, I. G., Costa, N. R. A., Guimarães, L. A., Mariano, F. N., Teixeira, S. C. P., \& Serrano, S. A. (2012). Acolhimentos de crianças e adolescentes em situações de abandono, violência e rupturas. Psicologia: Reflexão e Crítica, 25(2), 390-399. https://doi.org/10.1590/ S0102-79722012000200021

Salmún, S. L. (2014). Las adopciones de um niño. Cuestiones de Infancia, 16, 46- 57.

Sampaio, D. S., Magalhães, A. S., \& Féres-Carneiro, T. (2018). Pedras no caminho da adoção tardia: desafios para o vínculo parento-filial na percepção dos pais. Temas em Psicologia, 26(1), 311-324. doi: 10.9788/TP2018.1-12Pt

Santos, C. P., Fônseca, M. C. S. M., Fônseca, C. M. S. M. S., \& Dias, C. M. S. B. (2011). Adoção por pais solteiros: desafios e peculiaridades dessa experiência. Psicologia: Teoria e Prática, 13(2), 89-102.

Santos, C. M. C., Pereira, C. A. M. \& Nobre, M. R.C. (2007). A estratégia PICO para a construção da pergunta de pesquisa e busca de evidências. Revista Latino-americana de Enfermagem, 15(3), 1- 4.

Scorsolini-Comin, F., \& Santos, M. A. (2008). Aprender a viver é o viver mesmo: o aprendizado a partir do outro em um grupo de pais candidatos à adoção. Vinculo, 5(2), 101-219.

Sequeira, V. C., \& Stella, C. (2014). Preparação para adoção: grupo de apoio para candidatos. Revista Psicologia: Teoria e Prática, 16(1), 69- 78. https://doi. org/10.15348/1980-6906/psicologia.v16n1p69-78

Silva, M. L., \& Arpini, D. M. (2013). O impacto da nova Lei Nacional de Adoção no acolhimento institucional: o ponto de vista de psicólogos e assistentes 
sociais que integram as equipes técnicas. Psicologia em Revista, 19(3), 422-440.

Silva, C. L., \& Benetti, S. P. C. (2015). Older child adoption: a study of the affiliation process. Estudos de Psicologia, 32(1), 121-127. https://doi. org/10.1590/0103-166X2015000100011

Silva, F. H. O. B., \& Cavalcante, L. I. C. (2015). Rotinas familiares de crianças com necessidades especiais em família adotiva. Psicologia: Teoria e Pesquisa, 31(2), 173-180. https://doi. org/10.1590/0102-37722015021920173180

Silva, F. H. O. B., Cavalcante, L. I. C., \& Dell'Aglio, D. D. (2016). Pretendentes à adoção de crianças no Brasil: um estudo documental. Revista da SPAGESP, 17(2), 67- 80.

Silva, E. F. G., \& Santos, S. E. B. (2014). Paternidade adotiva: conjugando afetos consentidos. Revista da Abordagem Gestáltica, 20(2), 161-167.

Souza, M. L. N., Monteiro, C. A. S., \& Brito, L. M. T. (2016). A "nova cultura da adoção": reflexões acerca do cenário atual de adoção no Brasil. Dissertação de
Mestrado, Universidade Federal do Maranhão, São Luís, Brasil.

Speck, S., Queiroz, E. F., \& Martin-Mattera, P. (2018). Desafios da clínica da adoção: devolução de crianças. Estudos de Psicanálise, (49), 181-186.

Verceze, F. A., Silva, J. M., Oliveira, K. M., \& Sei, M. B. (2015). Adoção e psicoterapia familiar: uma compreensão winnicottiana. Revista da SPAGESP, 16(1), 92-106.

Vieira, G. A., Dotta, L. T., Farias, C., \& Henriques, M. R. (2014). The role of education in narrative identity construction of young people: focusing on young adoptees. Aletheia, 45, 9- 23.

Vieira, A. G., \& Henriques, M. R. (2013). A construção narrativa de identidade em jovens adotados: o caso de Beno. Análise Psicológica, 2(31), 145-157.

Weber, L. N. D (2004). Laços de ternura: pesquisas e histórias de adoção. ( $\left.3^{\mathrm{a}} \mathrm{ed}\right)$. Curitiba: Juruá.

Recebido em: 15/01/2018 Reformulado em: 25/10/2018 
Nota dos autores:

Apoio Financeiro: Capes (Coordenação de Aperfeiçoamento de Pessoal de Nível Superior

Sobre os autores:

Camila Aparecida Peres Borges é psicóloga e mestra em Psicologia pela Universidade Federal do Triângulo Mineiro, especialista em Psicologia Jurídica pela Universidade Cândido Mendes, pesquisadora do ORÍ - Laboratório de Pesquisa em Psicologia, Saúde e Sociedade (EERP-USP) e membro do Grupo Interinstitucional Pró-Adoção da cidade de Uberaba-MG.

ORCID: https://orcid.org/0000-0001-7419-8919

E-mail: camilaappborges@gmail.com

Fabio Scorsolini-Comim é psicólogo, doutor em Psicologia pela Faculdade de Filosofia, Ciências e Letras de Ribeirão Preto da Universidade de São Paulo, com pós-doutorado na área de Tratamento e Prevenção Psicológica. Professor do Departamento de Enfermagem Psiquiátrica e Ciências Humanas da Escola de Enfermagem de Ribeirão Preto da Universidade de São Paulo (EERP-USP). Docente colaborador do Programa de Pós-Graduação em Psicologia da Universidade Federal do Triângulo Mineiro (PPGP-UFTM). Coordenador do PROSA - Laboratório de Investigações sobre Práticas Dialógicas e Relacionamentos Interpessoais (UFTM-CNPq).

ORCID: https://orcid.org/0000-0001-6281-3371

E-mail: fabioscorsolini@gmail.com

Contato com os autores:

Rua Tenente Eurico, 128, CEP: 38072-090

Uberaba/MG - Bairro: Boa Vista

Telefone: (34) 992576034 - (34) 33222625 